\title{
Genetic and Molecular Dissection of Arsenic Hyperaccumulation in the
}

\author{
Banks, Jo Ann \\ Purdue University
}

\begin{abstract}
RESULTS TO DATE: We have constructed cDNA libraries from RNA isolated from arsenic treated gametophytes of the fern Pteris vittata. This library was made in a manner that allows each cDNA clone to be expressed in yeast. We have introduced this library into yeast cells, both wild type and arsensic sensitive mutants, and selected transformed yeast colonies with increased arsenic tolerance compared to the parental strains. From these screens we have identified putative homologs of the yeast ACR2 and ACR3 genes from Pteris vittata and, for the past year, have focused on characterizing the function of the ACR2 gene. In yeast, ACR2 is an arsenate reductase that is essential for arsenate tolerance. We refer to the Pteris vittata ACR2 gene as PVACR2. The deduced amino acid sequence of PVACR2 is highly similar to the yeast ACR2 and other related phosphatases.
\end{abstract}

To obtain purified protein, we have expressed and purified a recombinant form of the PVACR2 gene in E. coli. Purified PVACR2 protein has been used in in vitro enzyme assays to show that the protein has arsenate reductase activity; this activity requires glutathione and glutaredoxin as does the yeast ACR2 protein. Our results thus demonstrate that the PVACR2 protein is an arsenate reductase. We are currently determining the kinetic properties for this reaction. We are also assessing any potential phosphatase activity that this enzyme may have as, as phosphate and arsenate are analogs.

We predict that eliminating the expression of PVACR2 in Pteris gametophytes will alter their ability to reduce arsenate and, perhaps, affect their ability to tolerate or accumulate arsenic. To test this hypothesis, we will introduce into gametophytes by biolistic bombardment a construct that will systemically silence the endogenous PVACR2 gene, a method referred to as RNAi. We have demonstrated that this method works in Pteris gametophytes by targeting and silencing the magnesium chelatase gene from Pteris, a gene that is essential for chlorophyll biosynthesis in all plants. As expected, this resulted in colorless gametophytes. We have made a PVACR2 construct to target and silence the endogenous PVACR2 gene. These RNAi experiments are now underway.

In situ imaging and analysis of arsenic exposed P. vittata gametophytes has been performed using X-ray absorption spectroscopy (XAS), specifically X-ray absorption fluorescent spectroscopy (XAFS) and X-ray absorption near edge spectroscopy (XANES). This collaborative work was performed at SSRL (Stanford Synchrotron Radiation Laboratory) with Ingrid Pickering and Graham George. The results of XANES analysis in both P. vittata gametophytes and sporophytes indicates unchelated As(III) is the predominant arsenic species. XAFS imaging confirms the XANES analysis and suggests the As(III) is vacuolarly localized in the gametophyte. A manuscript describing these results has been written and will be submitted within the next few weeks.

Another question we have begun to address is oxidative stress in Pteris gametophytes. Our hypothesis is that Pteris is able to tolerate a high level of oxidative stress, and this may be related to its ability to tolerate and accumulate high levels of arsenic. Hallmarks of oxidative stress, namely serine acyl transferase (SAT) activity, O-acetyl serine (OAS), and glutathione (GSH) levels are being measured in gametophytes grown in different arsenic concentrations to test this hypothesis.

DELIVERABLES: Gumaelius, L., Lahner, B., Salt, D. and J. Banks. 2004. Arsenic hyperaccumulation in Pteris vittata gametophytes: A model system for analysis of arsenic tolerance and hyperaccumlulation. Plant Physiol. 136:3198-3209. 